\title{
REUNIÃO CLÍNICA INTERDISCIPLINAR - UMA ESTRATÉGIA DE ENSINO BASEADA EM PROBLEMAS NA GRADUAÇÃO EM FISIOTERAPIA: RELATO DE EXPERIÉNCIA
}

\author{
INTERDISCIPLINARY CLINICAL MEETING - AN EDUCATION STRATEGY BASED ON PRO- \\ BLEMS IN PHYSICAL THERAPY GRADUATION: EXPERIENCE REPORT
}

\author{
Susi Mary de Souza Fernandes \\ Ėtria Rodrigues \\ Zodja Graciani \\ Marcelo Fernandes \\ Gisela Rosa Franco Salerno \\ Denise Loureiro Vianna
}

Universidade Presbiteriana Mackenzie

\author{
Contato \\ Susi Mary de Souza Fernandes \\ E-mail: susifernandes@uol.com.br
}

\section{RESUMO}

Atualmente busca-se um modelo de ensino superior inovador, capaz de capacitar os profissionais para responder a demandas de saúde da sociedade. $\mathrm{O}$ Curso de Fisioterapia da Universidade Presbiteriana Mackenzie propõe nas diretrizes curriculares, consignadas ao Projeto Pedagógico Institucional e às Diretrizes Nacionais para Cursos de Graduação em Fisioterapia, um currículo no qual as disciplinas são oferecidas obedecendo a uma estrutura longitudinal de complexidade crescente e a metodologias ativas de ensino desenvolvidas em diferentes estratégias de ensino como: estudo de caso, solução de problemas e painel inseridos em todas as disciplinas do curso e concretizados no encontro de apresentação designado Reunião Clínica Interdisciplinar ( $\mathrm{RCl})$. A $\mathrm{RCl}$ tem-se constituído como uma ferramenta de motivação, instrumentalização e capacitação dos professores, proporcionado aos alunos o desenvolvimento de habilidades de argumentação, coerência e síntese, correlação teórico-prática, postura profissional crítica e reflexiva observadas, principalmente, nos alunos das últimas etapas.

Palavras-chave: Aprendizagem baseada em Problemas. Educação Superior. Fisioterapia.

\begin{abstract}
Nowadays, an innovative higher education model is sought, one that is able to train professionals to meet the health demands of society. The Physical Therapy course of Mackenzie University proposes the curriculum guidelines, assigned to the Institutional Educational Project and the National Guidelines for Physical Therapy in undergraduate courses, a curriculum in which courses are offered obeying a longitudinal structure of increasing complexity and active methodologies of teaching, developed in different teaching strategies such
\end{abstract}

as case study, problem solving and panel inserted in all course subjects and implemented in the meeting presentation called Interdisciplinary Clinical Meeting (RCl). $\mathrm{RCl}$ has been constituted as a tool for motivation, instrumentation and teacher capacitation, and provides students with the development of reasoning skills, consistency and synthesis, practical theoretical correlation, critical and reflective professional posture observed mainly in the students at the last stages.

Keywords: Problem-Based Learning. Education, Higher. Physical Therapy Specialty. 


\section{CONTEXTUALIZAC̣ÃO}

A apresentação curricular tradicional dos cursos de graduação está baseada na divisão de disciplinas e resulta na compressão de informações nos primeiros anos de curso, com sobrecarga do cognitivo e pulverização do conhecimento. Um dos principais problemas desse método é a falta de integração entre as disciplinas, principalmente entre as áreas básicas e as específicas.

Segundo o art. 52. da Lei de Diretrizes e Bases (LDB), a universidade é uma instituição pluridisciplinar de formação dos quadros de profissionais de nível superior, de pesquisa, de extensão, de domínio e cultivo do saber humano ${ }^{1}$. É reconhecida a necessidade de mudança, e da renovação nos processos educativos atuais, tendo em vista a preocupação com a unificação do saber.

Nessa perspectiva, as universidades brasileiras devem sofrer algumas alterações, passando do modelo pluridisciplinar para o interdisciplinar ${ }^{2}$. Para tanto, será necessária a revisão dos processos de formação acadêmica, de modo a converter o ensino tradicional em um modelo que privilegie a construção do conhecimento ancorada pelas Diretrizes Curriculares Nacionais ${ }^{3}$.

Desde a década de 1950, currículos alternativos têm sido propostos, mas geralmente com pouco sucesso. Há, hoje, um consenso entre os educadores de que o aprendizado deveria ser mais centrado no aluno, com maior carga horária para atividades de pesquisa e de estudo. A partir da década de 1970, houve o reconhecimento da participação ativa do indivíduo na incorporação do conhecimento; a importância de sua experiência prévia e do uso desta experiência como elemento motivador para o aprendizado ${ }^{4,5,6}$.

Dentro desse contexto, as escolas, principalmente, nos cursos de saúde, têm-se apoiado nas metodologias problematizadoras para alcançar tais objetivos. O primeiro movimento ocorreu nos cursos de Medicina, motivados nas experiências de países como Canadá e Holanda, e por recomendação das Sociedades das Escolas Médicas para países da África, Ásia e América Latina. No Brasil, essa metodologia estendeu-se, além da medicina, para os cursos de Enfermagem no Rio de Janeiro, Minas Gerais, São Paulo e Paraná que vêm incorporando a metodologia da solução de problemas em suas atividades curriculares normais e especiais ${ }^{4,5,7,8}$.

O Problem Based-Learning (PBL), difundido amplamente nas escolas de medicina, está baseado no modo de aprendizado do adulto e na compreensão do funcionamento da memória humana. Apresenta quatro objetivos básicos, a saber: a) a moti- vação para o aprendizado; b) o desenvolvimento do raciocínio clínico; c) a estruturação do conhecimento em contexto clínico; e d) o desenvolvimento de habilidades de autoaprendizado ${ }^{9}$. Impõe a criação de grupo tutorial, formado por um tutor e oito a dez alunos, que serão apresentados a uma situação-problema pré-elaborada, como estratégia de atuação ${ }^{10}$.

Outra estratégia baseada em solução de problemas é a Metodologia da Problematização, relatada por Bordenave e Pereira apud Berbel ${ }^{10}$. Foi definida por Charles Maguerez como Método Arco. O método se apresenta dividido em cinco etapas, a especificar: a) observação da realidade; b) pontos-chave; c) teorização; d) hipóteses de solução; e por último, e) aplicação à realidade (prática). Vale ressaltar que nessa metodologia os casos são estudados a partir da realidade ou de um recorte da realidade ${ }^{10,11}$.

As metodologias problematizadoras despontam como práticas educativas, no qual o principal fundamento é a participação ativa do aluno na construção do seu conhecimento ${ }^{3}$. Propiciando desenvolvimento operacional da atividade do estudante, de suas práxis, nela o estudante é motivado a buscar o conhecimento que o conduzirá na direção da solução do problema. Aparece como boa estratégia quando o objetivo é vincular o estudante a uma área profissional, uma vez que estimula ou amplia a significação dos elementos apreendidos em relação à realidade profissional ${ }^{5,12}$.

Tal modelo aparece como uma boa perspectiva para os cursos de saúde tendo em vista as prerrogativas do Ministério da Educação, apresentadas nas Diretrizes Curriculares Nacionais $(D C N s)^{13}$, e nas exigências atuais para formação dos profissionais de saúde para atender à demanda apresentada pelas novas diretrizes do Sistema de Saúde Brasileiro².

O profissional de saúde deve apresentar competências concretizadas em conhecimentos, habilidades e atitudes que o qualifique para atender às demandas de saúde da sociedade. Portanto, as práticas de ensino devem ser planejadas no contexto da realidade, no qual a prática de ensino e a dinâmica do serviço de saúde estejam articuladas em todos os momentos ${ }^{3}$.

Muito se tem discutido sobre mudanças na estruturação da educação superior em saúde. Vários processos de mudanças estão sendo desencadeados, porém as estratégias e métodos variam muito entre as universidades, o que pode ser justificado pela dificuldade individual e/ou coletiva na modificação de paradigmas. Para concretizar um projeto de ensino ativo, muitas estratégias de mobilização, instrumentalização e capacitação entre os envolvidos devem ser realizadas, o que demanda tempo e, sobretudo, anseio por conta dos envolvidos ${ }^{14}$. 
Segundo Feuerwerker ${ }^{6}$, na abordagem metodológica ativa, o currículo deve ser integrado, para que vários conteúdos possam se articular para atender à demanda de uma situação ou de um problema, de modo que a abordagem deve ser interdisciplinar. Para Gattás ${ }^{15}$, o processo de integração entre as disciplinas nos cursos de saúde é fundamental para formar profissionais mais engajados na realidade e nos processos de transformação dos sistemas de saúde.

As metodologias de ensino baseadas na problematização são práticas educativas nas quais o principal fundamento é a participação ativa do estudante na construção do seu conhecimento, sendo, portanto, consideradas como uma boa alternativa ao modelo tradicional no ensino das profissões de saú$d^{6}$. Ainda são poucas as escolas, em outras áreas da saúde, que conseguem implantar um currículo transformador, crítico e reflexivo, com adoção plena de metodologias problematizadoras ${ }^{3}$.

Diante dessa perspectiva, o Curso de Fisioterapia da Universidade Presbiteriana Mackenzie (UPM) propõe em suas diretrizes curriculares, consignadas ao Projeto Pedagógico Institucional e às DCNs para cursos de graduação em Fisioterapia ${ }^{13}$, um currículo estruturado em quatro grandes eixos, a saber: Ciências Biológicas e Saúde, Ciências Sociais e Humanas, Conhecimentos Biotecnológicos e Conhecimentos Fisioterapêuticos. Nele, as disciplinas ofertadas obedecem a uma estrutura longitudinal de complexidade crescente que propõe a integração contínua do conhecimento e a adoção de situações-problema. Para tanto, diferentes ferramentas pedagógicas são empregadas, como, por exemplo, estudo de caso, solução de problemas e painéis inseridos em todas as disciplinas do curso desde as primeiras etapas e concretizados no encontro de apresentação designado Reunião Clínica Interdisciplinar (RCl).

Dessa forma, o presente artigo tem como objetivo relatar a experiência na integração entre as diretrizes curriculares e as estratégias de ensino baseadas na problematização na perspectiva da formação profissional em fisioterapia como um processo contínuo e inter-relacionado, que favorece o acompanhamento da evolução do aluno na aquisição das competências e habilidades.

\section{DESCRIÇÃO DA EXPERIÊNCIA}

\section{Premissas para Implantação de Metodologias Ativas de Ensino na Graduação em Fisioterapia da UPM}

A partir da ampla reflexão sobre as DCNs13, buscou-se elaborar uma estrutura curricular que relacione dialeticamente as dimensões teoria e prática, direcionadas para a construção do conhecimento e protagonismo do aluno do Curso de Fisioterapia durante o processo de formação acadêmica; na perspectiva de formar um profissional fisioterapeuta com formação generalista, humanística, crítica e reflexiva, com capacidade de atuar em todos os níveis de atenção à saúde, com base no rigor científico e intelectual. Ademais, deter visão ampla, global e respeitar os princípios éticos e culturais do indivíduo e da coletividade, com capacidade para empreender e gerenciar serviços de Fisioterapia.

Nesse contexto, metodologias ativas de ensino foram elaboradas para facilitar e contribuir efetivamente com o processo ensino e aprendizagem, de modo que, ao participar de situações concretas, o alunado se reconheça como sujeito participativo da realidade.

Desde 2011, a principal estratégia pedagógica adotada no Curso de Fisioterapia da UPM, objetivando situações-problema, concretiza-se na forma de RCls. Trata-se da releitura de práticas clínicas correntemente adotadas em estágios curriculares, na prática profissional, e aqui expandidos a todos as etapas do Curso.

Os alunos das últimas etapas do Curso de Fisioterapia estão em fase de estágio supervisionado, no qual exercem a prática profissional, com objetivo de exercitar conteúdos teóricos e práticos desenvolvidos 
em etapas anteriores, porém com casos clínicos reais; divididos em quatro grandes áreas de atuação, assim distribuídas: Saúde Coletiva, Neurologia, Musculoesquelética e Hospitalar. Em cada uma dessas áreas, o aluno acompanhado de um professor supervisor torna-se responsável por um número determinado de pacientes.

Nesse momento, o aluno deverá, segundo objetivos traçados no Projeto Pedagógico, ser capaz de: a) Criar, propor e recomendar terapêuticas nas diferentes áreas de atuação da fisioterapia de modo humanizado; b) Justificar sua terapêutica baseado na integralidade; e c) Atuar criticamente diante dos princípios das ciências e da biotecnologia. Desse modo, são os principais atores na concretização da $\mathrm{RCI}$ tornando-se os responsáveis por eleger e conduzir um caso clínico real que será liderado por eles e trabalhado pelos alunos nos demais semestres.

Para concretização dos objetivos propostos para $\mathrm{RCl}$, várias estratégias metodológicas são executadas de acordo com o semestre letivo em que o aluno se encontra, obedecendo a seguinte sequência:

a) ESTUDO DE CASO: os alunos das últimas etapas em estágio são orientados a eleger um caso clínico, entre os pacientes atendidos, para realizar um estudo completo, desde a análise e interpretação da doença, levantamento de hipóteses, crítica às técnicas disponíveis para o tratamento, planejamento das estratégias de tratamento, prognóstico e repercussões socioambientais do quadro apresentado; de modo que, ao final, sejam capazes de traçar e apresentar uma proposta de solução na RCl. Após eleição do caso e decisão terapêutica, os alunos são orientados a disponibilizar o resumo do caso a todos os professores do curso.

b) SOLUÇÃO DE PROBLEMAS: os professores recebem o resumo e são orientados a trabalhar o caso nas aulas teóricas, relacionando o conteúdo do problema apresentado com a disciplina ministrada, independentemente da etapa que a disciplina se encontre, de tal modo que os alunos sejam capazes de exercitar pensamento reflexivo, crítico e criativo, correlacionando os conteúdos teóricos desenvolvidos na aula com a aplicação clínica apresentada no caso, a fim de reunir elementos para discussão posterior com os estagiários.

c) PAINEL: em dia predeterminado, todos os alunos do Curso reúnem-se em evento designado $\mathrm{RCl}$. Os alunos estagiários das últimas etapas apresentam o caso clínico estudado. Após a apresentação, com duração de 50 minutos, é aberta a sessão de debates. Nessa fase, os professores das diferentes áreas do curso, professores de outros cursos da saúde da universidade, bem como a plateia apresentam suas considerações aos alunos expositores. Estes respondem às argumentações; e, ao final, o professor supervisor de estágio, responsável pela área, realiza as considerações finais alinhando todos os pontos de vista.

As $\mathrm{RCls}$ acontecem semestralmente, e o número de casos apresentados podem variar de acordo com a distribuição das áreas de estágio. De tal modo que em cada $\mathrm{RCl}$ poderão ser discutidos de um a dois casos de diferentes áreas de estágio. 


\section{RESULTADOS E IMPACTOS}

$\mathrm{O}$

Quadro 1 apresenta os temas discutidos no pe-

ríodo que compreende o ano de 2011, início do

projeto, até o segundo semestre de 2015 , e ilus-

tra a abrangência da estratégia.

QUADRO 1. Áreas de estágios supervisionados contempladas nas RCIs e temas apresentados no período de 2011 a 2015
Semestre
Área de estágio
Tema

1\%/2011 MUSCULOESQUELÉTICA Intervenção Fisioterapêutica no paciente com Fratura da cabeça do rádio e Endoprótese: Estudo de Caso

NEUROLOGIA

Avaliação e Intervenção no paciente com Esclerose Múltipla: do Diagnóstico ao Prognóstico

\begin{tabular}{|c|c|c|}
\hline $2 \% / 2011$ & MUSCULOESQUELÉTICA & $\begin{array}{l}\text { Fisioterapia Aquática em pacientes com Fibromialgia e Lúpus Eritematoso Sistêmico: } \\
\text { Atividades em grupo }\end{array}$ \\
\hline \multirow[t]{2}{*}{$1 \% 2012$} & HOSPITALAR & Intervenção Fisioterapêutica no paciente com Doença Pulmonar Obstrutiva Crônica (DPOC) \\
\hline & SAÚDE COLETIVA & Intervenção Fisioterapêutica no Equilíbrio em Idosos \\
\hline \multirow[t]{2}{*}{$2 \% / 2012$} & HOSPITALAR & $\begin{array}{c}\text { Intervenção Fisioterapêutica em paciente com Síndrome da Membrana Hialina (SMH): } \\
\text { Estudo de Caso }\end{array}$ \\
\hline & SAÚDE COLETIVA & $\begin{array}{c}\text { Avaliação e intervenção na prevenção de doenças musculoesqueléticas de Trabalhadores } \\
\text { de um setor administrativo }\end{array}$ \\
\hline \multirow[t]{2}{*}{$1 \% / 2013$} & HOSPITALAR & Intervenção Fisioterapêutica em paciente com Insuficiência Cardíaca \\
\hline & SAÚDE COLETIVA & $\begin{array}{l}\text { Perfil de idosos institucionalizados e estratégias de intervenção na } \\
\text { Prevenção da Fragilidade }\end{array}$ \\
\hline $2 \% / 2013$ & SAÚDE COLETIVA & $\begin{array}{c}\text { Estratégias de Promoção a Saúde na Atenção Básica: } \\
\text { Aplicação de protocolo de avaliação para Ler/Dort segundo classificação internacional de } \\
\text { funcionalidade (CIF) }\end{array}$ \\
\hline
\end{tabular}

\begin{tabular}{lcr}
\hline $1 \% / 2014$ & SAÚDE COLETIVA & Intervenção Fisioterapêutica em mulheres com Incontinência Urinária Feminina \\
\hline $2^{\circ} / 2014$ & HOSPITALAR & Atuação da Fisioterapia no doente crítico - da UTI a alta \\
\hline $1 \% / 2015$ & NEUROLOGIA & Intervenção Fisioterapêutica em paciente com Mielomeningocele: um estudo de caso \\
\hline
\end{tabular}

2\%/2015 MUSCULOESQUELÉTICA Protocolo de Atendimento no paciente com politrauma - Repercussões e Prognóstico

Observa-se no Quadro 1 que as quatro grandes áreas de estágio foram contempladas. A distribuição e escolha das áreas contempladas em cada $\mathrm{RCl}$ é realizada de modo que todos os alunos estagiários possam experimentar essa prática, independentemente da área de estágio em que se encontrem. Entende-se que tornar o aluno ator principal na concretização da $\mathrm{RCl}$ contribui significativamente para a sua formação, atendendo às diretrizes traçadas no Projeto Pedagógico. 
Muitos são os benefícios que a $\mathrm{RCl}$ tem produzido no curso. Essa prática vem se configurando como importante estratégia para atender aos objetivos traçados no Projeto Pedagógico do Curso de Fisioterapia da UPM no que concerne ao desenvolvimento de práticas acadêmicas que assegurem experiências funcionais de aprendizagem, nas quais o alunado tem oportunidade de participar efetivamente de situações reais ${ }^{5,8}$.

Para os alunos, as repercussões têm sido notadas no desenvolvimento precoce de habilidades como capacidade de argumentação, coerência e síntese. Além disso, destacam-se a correlação teórico-prática e a postura profissional crítica e reflexiva observadas, principalmente, nos alunos das últimas etapas. Estes discutem ciência na visão do cotidiano, uma vez que o objeto estudado parte do contexto vivido, e, portanto, pode ser repensado, refletido e teorizado a partir da relação profissional-paciente, com o intuito de obter hipóteses de solução que atendam e alterem a realidade do paciente ${ }^{16}$.

Do mesmo modo, os alunos das primeiras etapas experimentam o contato precoce e amplo com os componentes curriculares inerentes a sua formação, o que permite uma visão diferenciada das disciplinas básicas, pois oferece sentido ao conteúdo desenvolvido desde as disciplinas básicas até as mais complexas; além de propiciar um olhar diferenciado para o binômio saúde-doença, que abandona a fragmentação do indivíduo proposta no modelo biomédico curativista para a adoção de um modelo que estimula o desenvolvimento da cidadania, possibilitando a compreensão do ser humano socialmente inserido ${ }^{16}$.

Outro aspecto importante refere-se à integração e à interdisciplinaridade entre os alunos das diversas etapas do curso de Fisioterapia, bem como de outros cursos de saúde da universidade, que interagem com o grupo que está apresentando o caso. Desde 2014, os professores e alunos do Centro de Ciências Biológicas e da Saúde, unidade acadêmica da universidade, no qual o Curso de Fisioterapia está inserido, são convidados a participar da $\mathrm{RCl}$. Os professores dos Cursos de Educação Física, Farmácia, Nutrição e Psicologia participam na qualidade de banca de arguição com objetivo de expor o ponto de vista da sua área de conhecimento, as possíveis intersecções e repercussões no quadro exposto, na perspectiva de que os alunos desses cursos, que participam como ouvintes, apropriem-se do caso e da importância do olhar clínico inovador, holístico e humanizado.

Com essa experiência, observa-se também uma mudança nos professores, que se apresentam mais motivados e em busca de novas perspectivas. Paulo Freire ${ }^{17}$ relata que quanto mais o professor possibilitar aos estudantes perceberem-se como seres inseridos no mundo, tanto mais se sentirão desafiados a responder aos novos desafios.

Embora, teoricamente, a aprendizagem baseada em problemas e a problematização se constituam de maneira distinta, ambas contribuem para mudança no processo de ensino-aprendizagem no ensino superior uma vez que modificam a forma tradicional de ensinar e aprender por estimular o protagonismo estudantil e a troca de saber ${ }^{5}$. Independentemente do modelo eleito, as metodologias ativas favorecem a aquisição e apropriação do conhecimento a partir da prática, na experiência vivenciada e percebida pelo indivíduo como problema. Segundo Schaurich et al.16, a aplicação de metodologias ativas no ensino em saúde suprime a fragmentação teoria-prática, uma vez que a solução do problema vivenciado encaminha para busca de solução no conteúdo teórico.

\section{DESAFIO NA CONSTRUÇÃO DE METODOLOGIAS ATIVAS}

A pesar dos inúmeros benefícios, alguns desafios devem ser vencidos, como, por exemplo, romper com as estruturas conservadoras que ainda hoje orientam os rumos da universidade. $O$ ensino em saúde em sua integralidade não permite a fragmentação, e, portanto, parte-se de uma visão que supõe aproximar a interface entre a diversidade das disciplinas que compõe a grade curricular.

Nesse sentido, a construção de um currículo integrador capaz de atender ás demandas do cenário atual, no qual a interação teórico-prática aconteça desde as primeiras etapas, nem sempre é possível de ser realizada. Percebe-se na carência de suporte apropriado do corpo acadêmico, institucional e no preparo dos próprios alunos algumas dificuldades para implementação das metodologias ativas.

Os professores da área da saúde apresentam-se resistentes. Geralmente, referem não dominar metodologias pedagógicas e apoiam-se na descrição de que são "profissional-professor". Argumentam não ter formação na licenciatura e que desenvolveram a técnica de ensino com base no exemplo de um antigo professor inspirador.

Segundo De Sordi ${ }^{18}$, o modelo de educação em saúde está em crise. Os alunos apresentam-se desinteressados, os professores encontram-se fixados no modelo tradicional de ensino, por sentirem-se despreparados metodologicamente para outras formas de ensinar e, além disso, resistem à mudança no modo de ensinar permanecendo fixados na racionalidade técnica, no modelo de saúde hospitalocêntrico e na centralização no saber do professor ${ }^{19}$. 
No entanto, com as exigências atuais de uma sociedade submetida a um processo de mudança acelerada, algumas escolas de saúde começam a aderir à metodologia ativa como estratégia de ensino. Já é comum encontrar cursos inteiros, ou algumas disciplinas, que aderiram a essa proposta para cursos de saúde $e^{4,5,7,8,16,18}$.

Tal fato, deve estar diretamente relacionado com um novo modelo de atenção à saúde que tem sido proposto. Este exige mudanças no sistema de formação dos profissionais e, consequentemente, nos projetos curriculares que devem ser integrados e que poderá levar à formação de profissionais mais comprometidos com a realidade de saúde e com sua transformação ${ }^{20}$.

Embora algumas escolas estejam aderindo a esse modelo, um outro desafio refere-se à mudança na postura do aluno, visto que a formação tradicional acompanha o estudante ao longo de toda sua formação. No ensino superior, as metodologias ativas apresentam-se para eles como um desafio extremo, que nem sempre estão preparados para assumir.

Marin et al. ${ }^{21}$ realizaram um estudo sobre metodologias ativas com estudantes de medicina. Os resultados revelaram que os alunos sentem que ocorreu uma mudança abrupta do método tradicional para as metodologias ativas que gera um sentimento de confusão na busca de conhecimento, principalmente em disciplinas básicas. Os estudantes relataram insegurança, grande esforço e necessidade de mudança de comportamento, maturidade e organização.

De tal modo que a prática da metodologia ativa, quando aplicada à formação de profissionais da saúde, exige um esforço intenso dos envolvidos, professor, instituição e alunos; a fim de respeitar a individualidade do ser humano, suas concepções e contemplar todas as dimensões do processo saúde-doença, para, com isso, atender às demandas do sistema de saúde brasileiro permitindo maior aproximação entre a teoria e a prática, entre as ações curativas e preventivas, entre a educação e o mercado de trabalho, tanto em uma perspectiva individual quanto coletiva.

\section{CONSIDERAÇÕES FINAIS}

A utilização integral de metodologias ativas é bastante complexa. Embora possibilite visualizar as diferenças entre as disciplinas, as correlações de forças entre as especialidades e o potencial que se agrega, quando comparada a práticas em que cada disciplina cuida de seu próprio conteúdo, apresenta-se mais trabalhosa.

Exige do professor uma mudança de postura para o exercício de um trabalho reflexivo com o aluno, que pressupõe disponibilidade do professor de pesquisar, de acompanhar e colaborar para o aprendizado crítico do estudante, o que frequentemente coloca o professor diante de situações imprevistas, novas e desconhecidas, exigindo que professores e alunos compartilhem de fato o processo de construção do conhecimento.

Ainda são poucas as experiências de implementação de propostas curriculares com adoção de metodologias ativas para os Cursos de Fisioterapia. Todavia, nessa experiência, notou-se que é possível a adoção com sucesso com base na observação da realidade e na reflexão crítica sobre as ações dos sujeitos, de forma a exercitar os conteúdos teóricos em conexão total com a prática. 


\section{Referências}

1. Brasil. Lei de Diretrizes e Bases da Educação Nacional. Lei número 9.394, 20 de dezembro de 1996.

2. Rossi CMS. Proposta de Interdisciplinaridade na Universidade. São Paulo: Papirus, 1995.

3. Vogt MSL. Os princípios andragógicos no contexto do processo ensino-aprendizagem da Fisioterapia. Tese de Doutorado em Ciências da Saúde, para obtenção do título de Doutor em Ciências da Saúde, da Faculdade de Ciências da Saúde, da Universidade de Brasília; Brasília-DF, 2007. Disponível em <http://repositorio.bce.unb.br/bitstream/10482/5300/1/TESE\%20-\%20Maria\%20Saleti\%20Lock\%20Vogt. pdf $>$. Acessado em 10 abr 2012.

4. Souza NR, Verdinelli MA. Aprendizagem ativa em Administração: um estudo da aprendizagem baseada em problemas (PBL) na graduação. PRETEXTO 2014; 15: 29 - 47.

5. Gomes MPC. et al. O uso de metodologias ativas no Ensino de graduação nas ciências sociais e da saúde - avaliação dos estudantes. Ciência \& Educação 2010; 16(1): 181-198.

6. Feuerwerker LA construção de sujeitos no processo de mudança na formação dos profissionais de saúde. Divulgação em Saúde para Debate 2000; 22: 18-24.

7. Maia JA. O currículo do ensino superior. In: Batista NA; Batista SH(Orgs.). Docência em saúde: tema e experiências. São Paulo: Ed. Senac, 101-133, 2004.

8. Zanolli MB. Metodologias ativas de ensino-aprendizagem na área clínica. In: Martins et al. Educação médica em transformação: Instrumentos para construção de novas realidades. São Paulo: HUCITEC, 40-61, 2004.

9. Toledo Júnior ACC, Ibiapina CC, Lopes SCF, Rodrigues ACP, Soares SMS. Aprendizagem baseada em problemas: uma nova referência para a construção do currículo médico. Revista Médica de Minas Gerais 2008; 18(2): 123-131.

10. Berbel NAN. A problematização e a aprendizagem baseada em problemas: diferentes termos ou dife rentes caminhos? Interface - Comunic, Saúde, Educ 1998; 2: 139-154.

11. lochida LC. Metodologias problematizadoras no ensino em saúde. In: Batista NA; Batista SH(Orgs.). Docência em saúde: tema e experiências. São Paulo: Ed. Senac, 153-166, 2004.

12. Anastasiou LGC; Alves LP. Estratégias de Ensinagem. In: Processos de Ensinagem na Universidade: pressupostos para as estratégias de trabalho em aula. 8 ed. Joinville/SC: UNIVILLE: 75-106, 2009.

13. Brasil. Ministério da Saúde. Conselho Nacional de Saúde. Diretrizes curriculares nacionais dos cursos de graduação em Fisioterapia, Fonoaudiologia e Terapia Ocupacional, 2002.

14. Zabit MN. Problem-based learning on student's critical thinking skills in teaching business education in malaysia: a literature review. American Journal of Business Education 2010; 3(6): 19-32.

15. Gattás MLB. Interdisciplinaridade: formação e ação na área da saúde. Ribeirão Preto: Holos Editora; 2006.

16. Schauric D, Beheregaray FC, Almeida MA. Metodologia da problematização no ensino em enfermagem: uma reflexão do vivido no PROFAE / RS. Rev Enferm 2007; 11(2): 318 - 24.

17. Freire $P$. Pedagogia da autonomia: saberes necessários à prática educativa. 31 . ed. Rio de Janeiro: Paz e Terra, 2005.

18. De Sordi MRL. Avaliação da aprendizagem universitária em tempos de mudança: a inovação ao alcance do educador comprometido. In: Veiga IP, Castanho MELM. (Orgs.) Pedagogia Universitária: a aula em foco. Campinas, SP: Papirus, 2000.

19. Colombo AA.; Berbel NAN. A Metodologia da Problematização com o Arco de Maguerez e sua relação com os saberes de professores. Semina: Ciências Sociais e Humanas 2007; 28(2): 121-146.

20. Abreu ES et al. Interdisciplinaridade, saúde e qualidade de vida no ambiente universitário: relato de experiência. Rev. Simbio-Logias 2014; 7(10): 107-118.

21. Marin MJS et al. Aspectos das fortalezas e fragilidades no uso das Metodologias Ativas de Aprendizagem. Rev Bras Educação Médica 2010; 34(1): 13 - 20. 